\title{
RICKETS DUE TO DIETARY CALCIUM DEFICIENCY IN MANCHESTER
}

\section{Fatema Zehra Juma1, Dr Rui Santos ${ }^{2}$, Professor Zulf Mughal² \\ ${ }^{1}$ Manchester Medical School, ${ }^{2}$ Royal Manchester Children's Hospital}

\begin{abstract}
BACKGROUND \& AIMS: Rickets is a childhood condition resulting from impaired mineralisation of the growth plate, resulting in bony deformities. A retrospective survey was undertaken to identify causes of rickets in children treated at the Royal Manchester Children's Hospital from 2009 to 2014.

METHODS: Cases of rickets were identified through a search of all paediatric radiology reports containing the words 'Rickets' or 'Osteomalacia' and confirmed with reference to relevant biochemical tests. Those with serum $25 \mathrm{OHD}$ concentrations $<25 \mathrm{nmol} / \mathrm{L}$ were classified as vitamin $\mathrm{D}$ deficiency rickets and those with $250 \mathrm{OHD}>50 \mathrm{nmol} / \mathrm{L}$ and a history of inadequate dietary calcium intake as calcium deficiency rickets.

RESULTS: Eighty cases of rickets were identified of which 69 patients had nutritional rickets. Four* children had rickets due to dietary calcium deficiency and the rest of the cases of nutritional rickets were primarily due to vitamin $D$ deficiency. Three of the cases with calcium deficiency rickets had cow's milk protein allergy and the $4^{\text {th }}$ disliked and avoided dairy products. The relevant biochemical data are shown in the table below.

CONCLUSIONS: Rickets due to dietary calcium deficiency has been reported in South Africa, Northern Nigeria, Bangladesh and parts of India. Whilst vitamin D was the commonest cause of nutritional rickets in our survey, we also identified 4 cases of rickets due to dietary calcium deficiency. This survey highlights the importance of providing adequate calcium intake, particularly in children with food allergies/intolerances.

* On further review of patient data after the abstract was submitted, there were 2 cases of calcium deficiency rickets, not 4
\end{abstract}

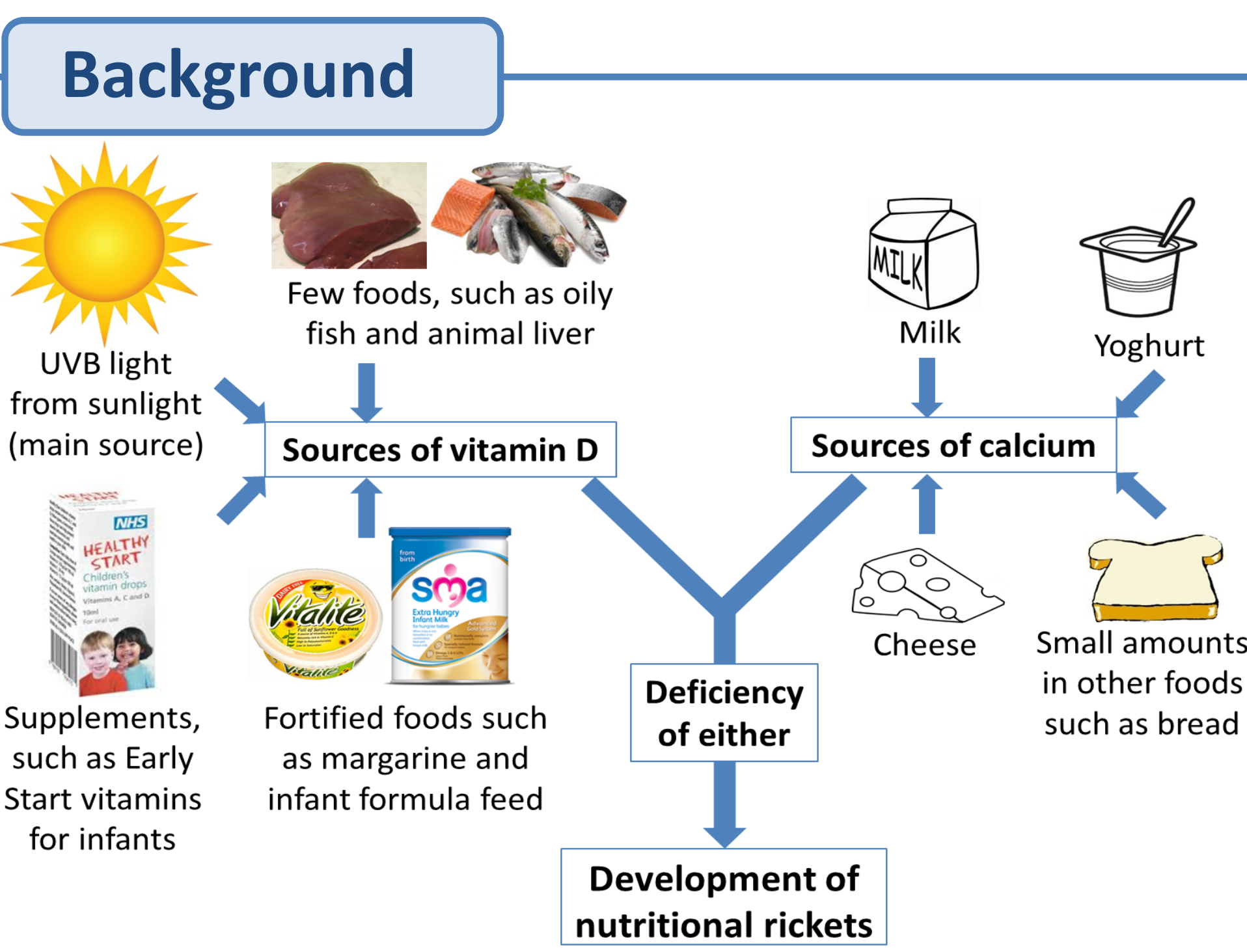

Rickets is a childhood condition characterised by impaired mineralisation of the growth plate and osteoid. This causes characteristic bony deformities in toddlers, as shown by the images on the left.

The most common cause of rickets is due to vitamin D deficiency. It can also sometimes occur due to calcium deficiency although this has mainly only been reported in developing countries. Recently, Goldacre et al ${ }^{1}$

found that the number of cases of rickets are increasing and are currently the highest they have been for 5 decades.

\section{Aims}

1. Determine how the number of cases of rickets seen at Royal Manchester Children's Hospital (RMCH) varied from 2009 to 2014

2. Identify the cause for these cases

3. Determine how many of the cases required admission

\section{Methods}

Searched radiology reports from 2009-2014

including the words 'Rickets' or 'Osteomalacia'

Radiographs reviewed

alongside biochemistry results

11 genetic cases of

69

Cases of nutritional rickets that had both:

\begin{tabular}{|c|c|c|}
\hline Radiological evidence & Biochem & den \\
\hline Cupping & $\downarrow / \leftrightarrow$ & Calcium \\
\hline Fraying & $\downarrow$ & Phosphate* \\
\hline Widening of metaphysis & $\uparrow$ & PTH \\
\hline
\end{tabular}

*Can be transiently raised due to PTH resistance.

Cases further subcategorised by cause of nutritional rickets

11 cases where cause could not be identified

\section{Cause of rickets}

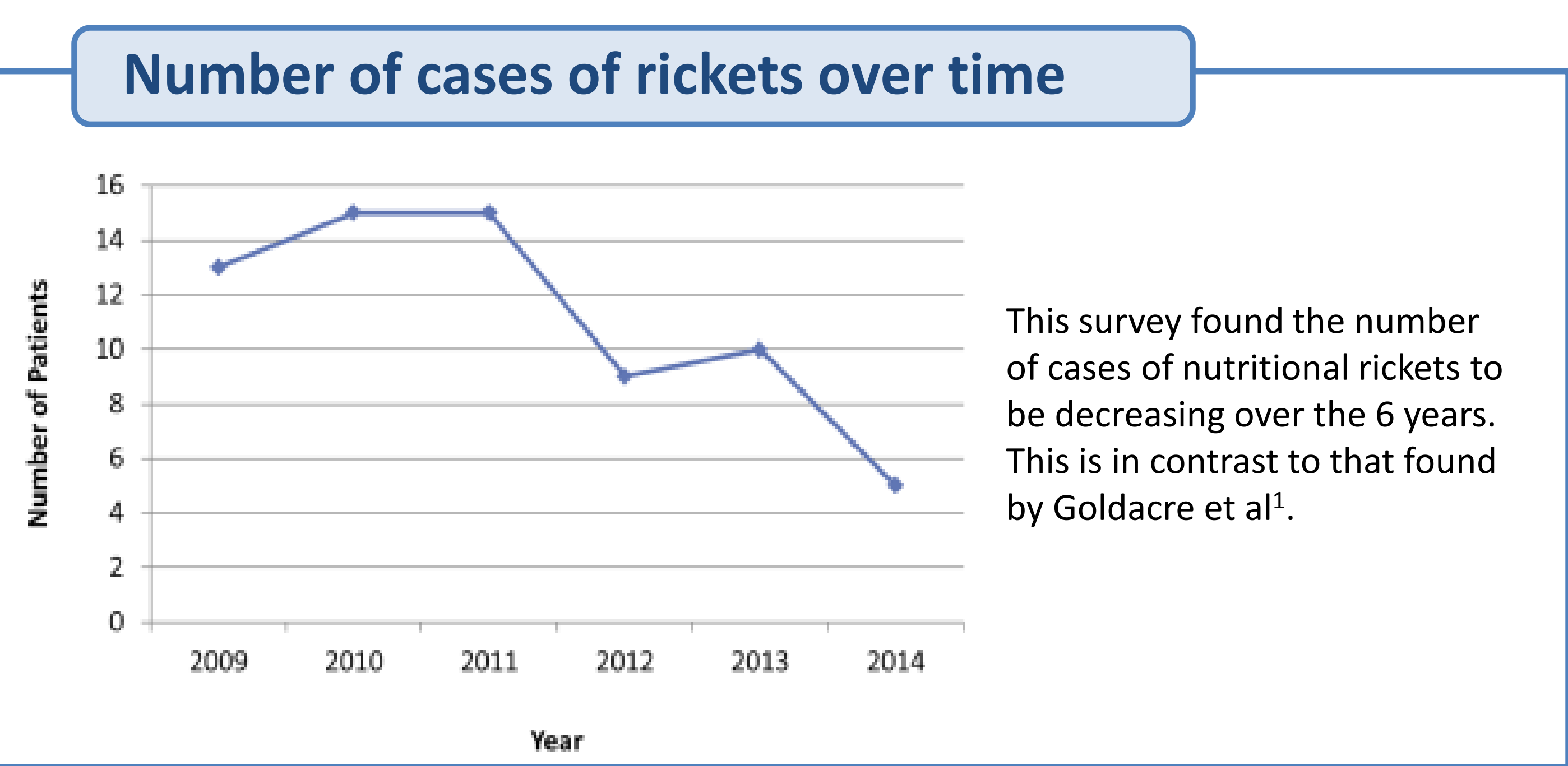

\section{Ca deficiency case 1}

25 month old Sudanese boy

- Presented with delayed walking

- Had allergies to cows milk, fish, egg

- Management:

- Dalavit $0.6 \mathrm{mls}$ OD

- Calcium (failed to take)

- Diet:

Breastfed since birth

Refused to drink soya milk

Weaning on rice, pasta, potatoes, chicken

- Florid rickets on examination

\section{Ca deficiency case 2}

$\mathbf{1 1}$ year old African boy

- Presented with bilateral genu-valgus

- Has focal seizures

Management:

Carbamazepine 100mgs BD

Not on any supplements

- Diet:

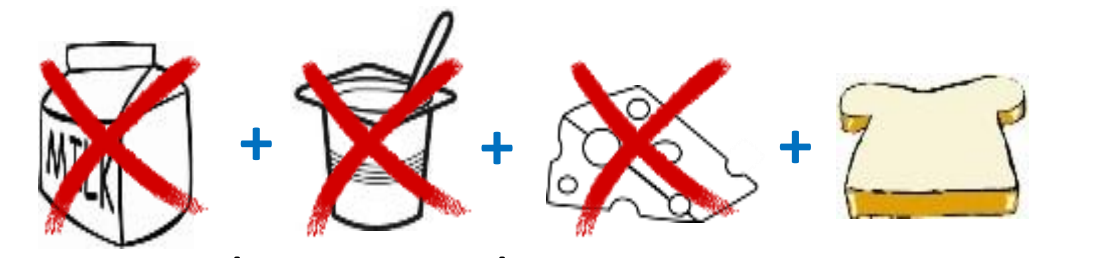

No milk/ yoghurt/ cheese, 2 slices bread

$=$ Estimated calcium intake $<200 \mathrm{mgs} /$ day (1/5 of his RDA!)

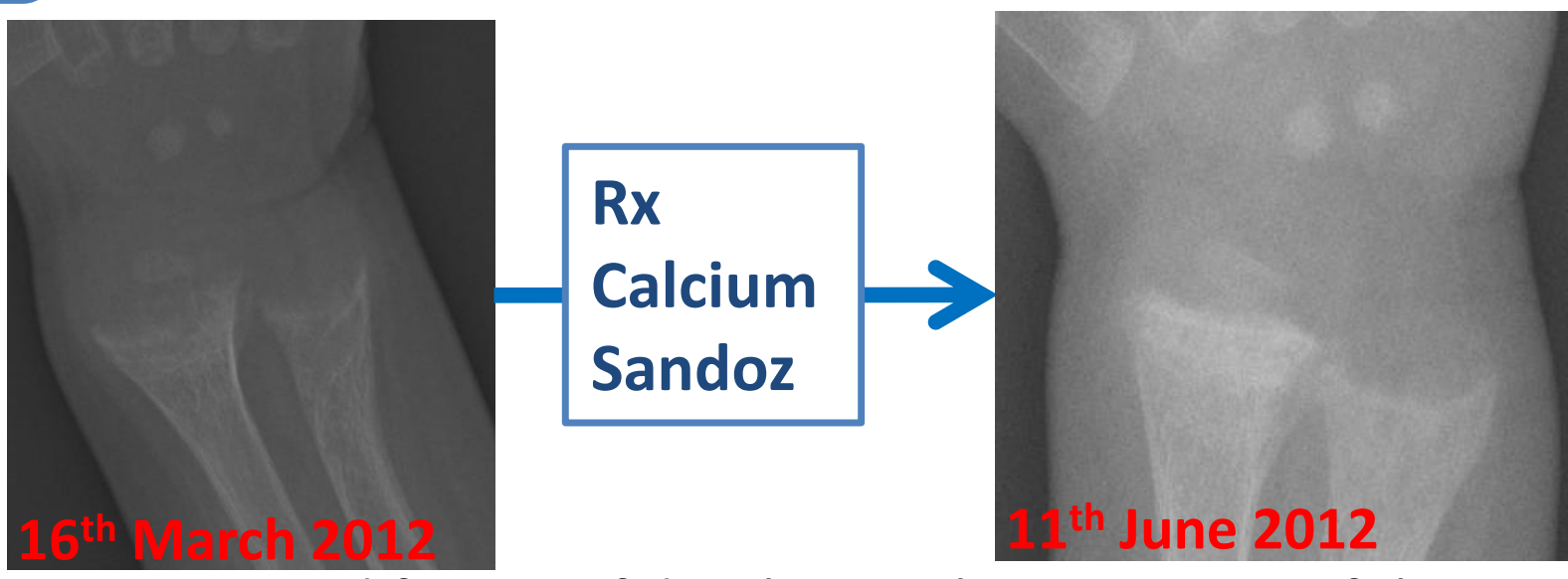

Cupping and fraying of distal metaphysis on $\mathrm{x}$-ray of the wrist, which improved after treatment

\begin{tabular}{|c|c|c|c|c|c|}
\hline & \multicolumn{2}{|c|}{ Case 1} & \multicolumn{3}{|c|}{ Case 2} \\
\hline $\begin{array}{l}\text { Corr Calcium } \\
(2.2-2.7 \mathrm{mmol} / \mathrm{L})\end{array}$ & 2.39 & $\rightarrow \quad 2.51$ & 1.97 & $\rightarrow$ & 2.41 \\
\hline $\begin{array}{l}\text { Phosphate } \\
\text { (1.05-1.95 mmol/L) }\end{array}$ & 0.79 & $\rightarrow \quad 1.50$ & 0.79 & $\rightarrow$ & 0.89 \\
\hline $\begin{array}{l}\text { ALP } \\
(60-300 \mathrm{U} / \mathrm{L})\end{array}$ & 1301 & $\rightarrow 180$ & 1898 & $\rightarrow$ & $2 !$ \\
\hline $\begin{array}{l}\text { PTH } \\
(10-60 \mathrm{pg} / \mathrm{ml})\end{array}$ & 465 & $\longrightarrow \quad 19$ & 865 & $\rightarrow$ & 31 \\
\hline $\begin{array}{l}\text { (OH)D } \\
0-70 \mathrm{nmol} / \mathrm{L})\end{array}$ & 70.6 & $\longrightarrow 78.6$ & 139 & $\rightarrow$ & 39.1 \\
\hline
\end{tabular}

\section{Cases requiring admission}

A third of patients with nutritional rickets were admitted for complications or comorbidities of their rickets and/or vitamin $D$, totalling at 123 days of admission.

Causes include:

- 8 Seizures

- 4 Fractures

- 2 Tetany

- 2 Surgeries to correct deformities

- 1 Dilated cardiomyopathy (37 days of admission)

- 1 Elective procedure cancelled due to rickets

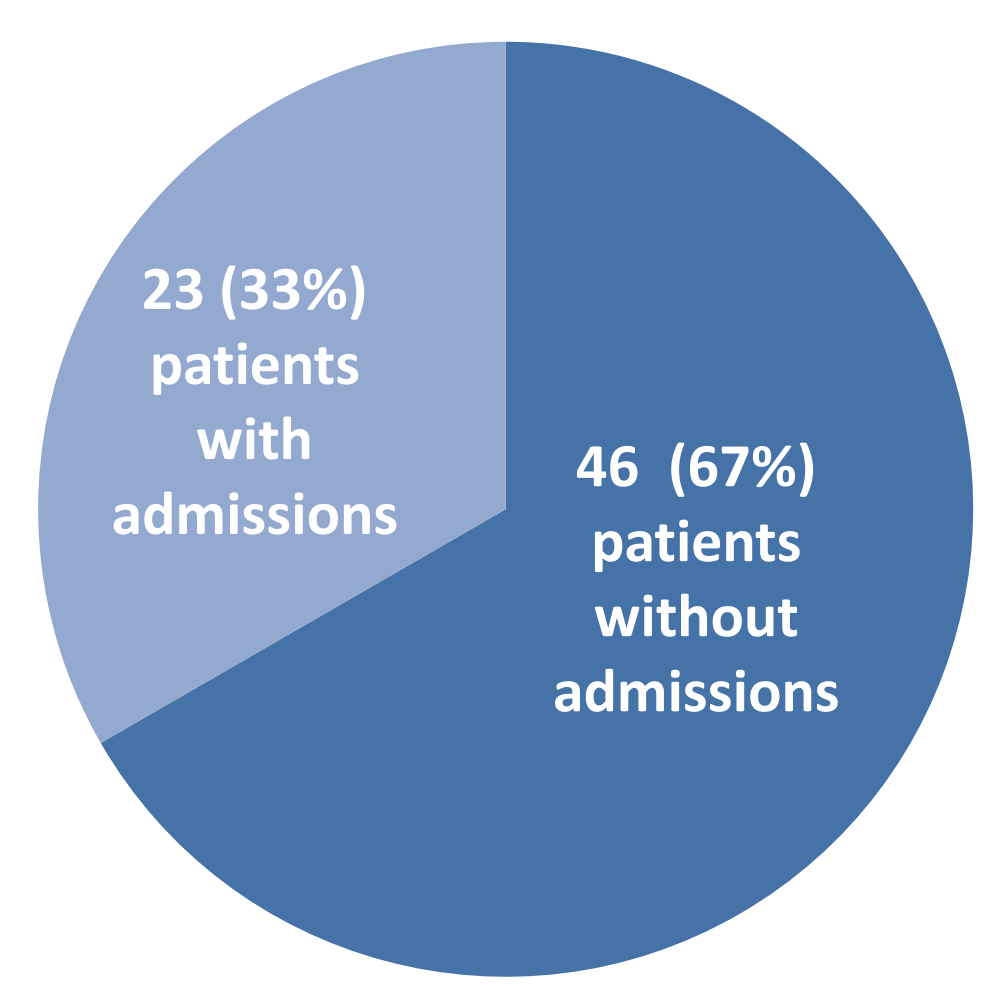

\section{Conclusions \& Recommendations}

- The number of cases of nutritional rickets at RMCH have been decreasing since 2009 .

- Vitamin D deficiency remains the most common cause of rickets.

Prevention via provision of vitamin D supplements is vital

Rickets due to dietary calcium deficiency exists in the UK.

Calcium supplements are important

- Rickets \& associated conditions are an important cause of hospital admissions

\section{Reference}

1. Goldacre M, Hall N, Yeates DG. Hospitalisation for children with rickets in England: historical perspective. The Lancet 2014; 383(9917):597-8.

\section{Please note}

There were 2 patients with calcium deficiency rickets, not 4 as stated in the previous abstract. This is on further review of the patient notes. 